\title{
Consumo de Estimulantes Cerebrais por Estudantes em Instituições de Ensino de Montes Claros/MG
}

\section{Consumption of Brain Stimulants by Students in Montes Claros/MG}

\section{PALAVRAS-CHAVE}

- Estimulantes Cerebrais.

- Estudantes.

- Metilfenidato.
Luiza Côrtes Santana ${ }^{1}(\mathbb{D}$ Andreza Neves Ramos ${ }^{\mathrm{I}}$ (D) Bruna Lopes de Azevedo ${ }^{\mathrm{I}} \mathrm{D}$ Inácio Luiz Morais Neves ${ }^{\mathrm{I}}$ (D) Mateus Magalhães Lima ${ }^{\mathrm{I}}$ (D) Marcos Vinícius Macedo de Oliveira ${ }^{\mathrm{I}} \mathbb{D}$

\section{RESUMO}

Introdução: A entrada dos jovens na universidade gera uma mudança de ambiente e costumes, tornando-se um período de grande vulnerabilidade para o uso de substâncias psicoativas. O objetivo deste estudo foi analisar o uso de substâncias psicoativas por estudantes de graduação e pré-vestibulandos, de Montes Claros-MG. Métodos: Foi realizado um estudo quantitativo, transversal, que avaliou 348 estudantes, em instituições de ensino prévestibular (52 estudantes) e superior (98 de Engenharia Civil, 68 de Medicina e 130 de Direito), da cidade de Montes Claros, em Minas Gerais, analisando fatores associados ao uso de psicoestimulantes. Para a obtenção dos dados, utilizou-se um questionário padronizado e validado de autopreenchimento. Obtiveram-se informações sobre o uso de metilfenidato (Ritalina), cafeína, pó de guaraná, modafinila, piracetam, energético, anfetamina e ecstasy. Resultados: Dos 348 estudantes entrevistados, cerca de 53,7\% faziam uso de algum psicoestimulante. Houve um maior uso de substâncias psicoativas nos participantes do grupo do pré-vestibular (75\%) em relação ao ensino superior (50\%). Observou-se uma maior prevalência do uso atual de cafeína (63,5\%) e de pó de guaraná (11,5\%) entre os estudantes de pré-vestibular, e de ecstasy (1,7\%) e cloridrato de metilfenidato (1,9\%) entre os estudantes de ensino superior. A redução do sono (64,9\%) foi o efeito mais percebido pelos usuários de estimulantes cerebrais do ensino superior, seguido de melhora na concentração (48\%), no bem-estar (45,3\%) $e$ no raciocínio (38,5\%), redução da fadiga (33,1\%), melhora na memória (23,6\%) e redução do estresse (23\%). Entretanto, nos estudantes do pré-vestibular apenas a melhora no raciocínio $(43,6 \%)$ e a redução do estresse (23\%) obtiveram relevância significativa. Conclusões: Foi possível observar uma maior prevalência do uso de psicoestimulantes nos pré-vestibulandos em relação ao grupo dos universitários. Entre as áreas do ensino superior, não foram encontradas diferenças quanto ao uso dos estimulantes cerebrais pesquisados. É preciso destacar os malefícios do uso de psicoestimulantes em longo prazo, sobretudo a dependência e a tolerância química. Em função disso, o apoio familiar e o psicopedagógico são indispensáveis para prevenir e tratar as consequências do uso desmedido de psicoestimulantes. 


\section{KEYWORDS}

- Brain Stimulants.

- Students.

- Methylphenidate.

\section{ABSTRACT}

Introduction: When young people enter the university, it generates a change of environment and customs, thus becoming a period of great vulnerability for the use of psychoactive substances. The objective of this study was to analyze the use of psychoactive substances in undergraduate and pre-college students, in Montes Claros-MG, associating the epidemiological profile, the frequency of use among the groups and describe the main benefits and side effects of the used substances. Methods: A quantitative cross-sectional study was carried out, which evaluated 348 students in pre-college (52 students) and higher education institutions (98 Civil Engineering, 68 Medical and 130 Law students), in the city of Montes Claros, state of Minas Gerais, analyzing factors associated with the use of psychostimulants. To obtain the data, a standardized and validated self-filling questionnaire was used. Information on the use of Methylphenidate (Ritalin ${ }^{\star}$ ), Caffeine, Guarana Powder, Modafinil, Piracetam, Energy drinks, Amphetamine and Ecstasy were obtained. Results: Of the 348 students interviewed, about 53.7\% used some type of psychostimulant. There was a greater use of psychoactive substances among the participants of the pre-college group (75\%) in relation to those of the higher education group (50\%). There was a higher prevalence of current use of caffeine (63.5\%) and guarana powder (11.5\%) among pre-university students, Ecstasy (1.7\%) and Ritalin (1.9\%) among students of higher education institutions. The reduction of sleep (64.9\%) was the most perceived effect by the users of brain stimulants in higher education, followed by improvement in concentration (48\%), wellness (45.3\%), reasoning (38.5\%), fatigue reduction (33.1\%), memory improvement (23.6\%) and stress reduction (23\%). However, among the pre-college students only the improvement in reasoning (43.6\%) and stress reduction (23\%) obtained significant relevance. Conclusion: It was possible to observe a higher prevalence of the use of psychostimulants among the pre-college students in relation to the group of university students. Among the areas of higher education, no differences were found regarding the use of the assessed brain stimulants. It is necessary to highlight the harmful effects of the use of psychostimulants in the long term, especially dependence and chemical tolerance. As a result, family and psycho-pedagogical support are indispensable to prevent and treat the consequences of the excessive use of psychostimulants.

Recebido em 20/11/19

Aceito em 6/12/19

\section{INTRODUÇ̃̃O}

A entrada dos jovens na universidade leva a uma mudança de ambiente e costumes, tornando-se um período de grande vulnerabilidade para o uso de substâncias psicoativas ${ }^{1}$. Os jovens que adentram as universidades possuem destaque no uso dessas substâncias ${ }^{2}$. Contudo, há relatos de que a fase de preparação para entrada na faculdade - cursinho pré-vestibular - é um período perturbador para os estudantes, pois há um ritmo diferente de estudos, levando a níveis maiores de estresse e exaustão ${ }^{3}$.

A extensa carga horária, a necessidade de estudos constantes, a preocupação e a cobrança do próprio aluno, em relação ao futuro e ao seu rendimento atuam como fatores que podem contribuir para o estímulo do uso de substâncias psicoativas. Observa-se uma maior necessidade de se manter mais tempo acordado para tentar suprir a demanda de estudos, o que propicia o uso de estimulantes do sistema nervoso central ${ }^{4}$.

Os estimulantes cerebrais são elementos que conseguem elevar o estado de vigília e estímulo, podendo também melhorar o humor, o desempenho cognitivo e a depressão ${ }^{5}$. São classificados como naturais obtidos pela extração vegetal, a exemplo da cafeína - e sintéticos - obtidos por meio de laboratórios, a exemplo do metilfenidato ${ }^{6}$.

Entre os estudantes, observa-se uma maior prevalência do uso da cafeína, cujo efeito é considerado tão fisiológico que o indivíduo pode ser levado à dependência sem perceber. Outro exemplo é o pó de guaraná, que possui como princípio a guaranina, um composto alcaloide que é processado mais lentamente no corpo, quando comparado à cafeína, mas que tem efeitos que podem durar horas ${ }^{6}$.

Já cloridrato de metilfenidato, também conhecido por Ritalina, é um dos estimulantes mais utilizados no mundo, com o objetivo principal de tratar o transtorno de déficit de atenção e hiperatividade (TDAH). Entretanto, o medicamento vem sendo utilizado de forma inadequada e para outros fins ${ }^{7}$.

A utilização descontrolada de psicoestimulantes por universitários que não possuem recomendação é uma questão que merece enfoque. Há poucos estudos realizados sobre essa prática no Brasil, o que incentiva uma busca mais aprofundada sobre os motivos que levam a esses hábitos indiscriminados ${ }^{8}$.

O presente trabalho buscou analisar o uso de estimulantes cerebrais por estudantes de graduação e pré-vestibulandos, de Montes Claros-MG.

\section{MÉTODOS}

Desenho do estudo

Foi realizado um estudo quantitativo, transversal, que avaliou estudantes, em instituições de ensino, pré-vestibular e superior, da cidade de Montes Claros, em Minas Gerais, para analisar fatores associados ao uso de psicoestimulantes. A escolha do cursinho e da instituição de ensino foi realizada por conveniência dospesquisadores, por conta da facilidade para a coleta das informações.

\section{População e amostra}

O universo dos participantes comporta 3.010 alunos; 600 acadêmicos do curso de Medicina, 1.300 Direito, 760 Engenharia civile 350 do pré-vestibular. O cálculo amostral com nível de confiança da pesquisa foi estimadoem $95 \% \mathrm{e}$ o erro amostral com margem de $5 \%$ indicou a investigação de 400 estudantes, número que foi superestimado para reduzir a margem de erro. $\mathrm{O}$ cálculo foi alcançado a partir da fórmula $n=N Z 2 p(1-p) e 2+Z 2 p(1-p)$, em que " $n$ " 
representa o tamanho amostra "N" o tamanho do universo a ser calculado; " $\mathrm{Z}$ " o desvio do valor médio que é aceito para alcançar o nível de confiança; "e" a margem de erro admitida; e " $p$ " a proporção que se espera encontrar. A amostra foi selecionada por meio de um sorteio de nomes na lista de matriculados dos estudantes de ensino superior por meio de lista de presença dos estudantes de cursinho pré-vestibular, comportando 296 estudantes de ensino superior (98 de Engenharia civil, 68 de Medicina e 130 de Direito) e 52 do pré-vestibular.

Entre os critérios de inclusão da pesquisa, os estudantes deveriam estar matriculados na faculdade ou no pré-vestibular e ter idade maior que 18 anos. Como critérios de exclusão, o indivíduo não poderia utilizar medicamentos psicoestimulantes sob prescrição médica, apresentar deficiência visual ou auditiva e/ou TDAH e narcolepsia, e preencher os formulários de pesquisa de forma incompleta ou inconsistente.

\section{Instrumentos e procedimentos}

Para a coleta de dados utilizou-se um formulário padronizado, de autopreenchimento e com questões objetivas. Essequestionário adaptado a partir do instrumento proposto pelo The smart drug study ${ }^{9}$, foi respondido pelos próprios estudantes. Trata-se de um formulário para coleta de dados das seguintes variáveis: sexo (feminino e masculino); rotina de estudos; tabagismo e/ou outras drogas; exercícios físicos; uso de suplementos para ganho de massa muscular; dieta para perda ponderal; horas de sono; qualidade do sono; uso de indutores do sono; uso de outras medicações contínuas; autoavaliação da saúde; pessoas que habitam a mesma moradia; uso atual ou passado de cafeína, energéticos, anfetaminas, metilfenidato e ecstasy; efeitos observados (no sono, estresse, fadiga, memória, raciocínio, concentração e bem-estar). Foram considerados usuários de psicoestimulantes apenas aqueles estudantes que informaram realizar o uso de pelo menos um dos estimulantes cerebrais apontados nos formulários durante o período de ocorrência da pesquisa.

No caso dos estudantes do pré-vestibular, redigiram-se questões específicas sobre o curso pretendido, o tempo no cursinho e outros cursos realizados. Aos universitários, dirigiram-se as seguintes variáveis: graduação, período do curso, se já foi reprovado, carga horária do curso, turno do curso e atividades extracurriculares.

O questionário foi aplicado no período compreendido entre fevereiro e março de 2019, durante aulas das turmas pesquisadas, e com a permissão do professor. Ao serem selecionados, os estudantes assinaram o Termo de Consentimento Livre e Esclarecido (TCLE) e, após, responderam ao questionário sob supervisão para esclarecimento de dúvidas, se houvesse.

\section{Análise dos dados}

Os dados obtidos foram tabulados no software Statiscal Package for the Social Sciences (SPSS), versão 22.0, com 95\% de confiabilidade $(\mathrm{p}<0,05)$. Adotaram-se testes qui-quadrado $\left(\mathrm{x}^{2}\right)$ e exato de Fisher para a comparação dos grupos de participantes em relação às variáveis investigadas. Houve comparação entre estudantes universitários e do pré-vestibular com o propósito de avaliar as variáveis do estudo. Além disso, houve comparação dentro do grupo de pré-vestibulandos, com suas variáveis específicas, bem como entre os universitários e suas variáveis específicas.

Aspectos éticos

A pesquisa foi aprovada para execução pelo Comitê de Ética em Pesquisa do Centro Universitário FipMoc (UniFIPMoc), seguindo os princípios éticos definidos pela Resolução nº 466/12 do Conselho Nacional de Saúde, para realização de pesquisas envolvendo seres humanos: Certificado de Apresentação para Apreciação Ética (CAAE) $\mathrm{n}^{\circ}$ 03913618.7.0000.5109, Parecer n 3.066.986, 2018.

\section{RESULTADOS}

Avaliaram-se 348 participantes, dos quais o sexo masculino representou $52,9 \%$. Além disso, a maioria dos estudantes não exerce nenhuma atividade laboral, não utiliza medicações para dormir e não faz uso de nenhuma medicação de uso diário. Entre os estudantes entrevistados, observouse ainda que a maior parte deles (53,7\%) fazia uso de algum tipo de psicoestimulante. Houve significância estatística $(\mathrm{p}=0,001)$ demonstrando maior uso de psicoestimulantespelosparticipantes do grupo do pré-vestibular (75\%) em relação ao ensino superior (50\%). Também foram identificadas associações significativas em relação ao uso de medicações diárias $(\mathrm{p}=0,010)$ e de medicações para dormir $(\mathrm{p}<0,001)$ (Tabela 1$)$.

\begin{tabular}{|c|c|c|c|}
\hline \multicolumn{4}{|c|}{$\begin{array}{c}\text { Tabela } 1 \\
\text { Análise das características e dos hábitos dos estudantes avaliados em } \\
\text { relação ao tipo de curso em andamento }\end{array}$} \\
\hline $\begin{array}{c}\text { Características e hábitos dos } \\
\text { estudantes }\end{array}$ & $\begin{array}{l}\text { Curso pré- } \\
\text { vestibular }\end{array}$ & Graduação & $\mathbf{P}$ \\
\hline Sexo & & & 0,452 \\
\hline Feminino & $27(51,9 \%)$ & $137(46,3 \%)$ & \\
\hline Masculino & $25(48,1 \%)$ & $159(56,7 \%)$ & \\
\hline Natural de Montes Claros & & & 0,073 \\
\hline Não & $35(67,3 \%)$ & $164(55,4 \%)$ & \\
\hline Sim & $17(32,7 \%)$ & $132(44,6 \%)$ & \\
\hline Reside & & & 0,253 \\
\hline Sozinho & $8(15,4 \%)$ & $33(11,1 \%)$ & \\
\hline Com alguém & $44(84,6 \%)$ & $263(88,9 \%)$ & \\
\hline Toma café da manhã? & & & 0,728 \\
\hline Não & $14(26,9 \%)$ & $73(24,7 \%)$ & \\
\hline Sim & $38(73,1 \%)$ & $223(75,3 \%)$ & \\
\hline Fuma ou já fumou? & & & 0,536 \\
\hline Não & $38(73,1 \%)$ & $228(77 \%)$ & \\
\hline Sim & $14(26,9 \%)$ & $68(23 \%)$ & \\
\hline Prática de exercício físico & & & 0,165 \\
\hline Não & $22(42,7 \%)$ & $96(32,4 \%)$ & \\
\hline Sim & $30(57,7 \%)$ & $200(67,6 \%)$ & \\
\hline $\begin{array}{l}\text { Uso de suplementos para } \\
\text { ganho de massa muscular }\end{array}$ & & & 0,501 \\
\hline Não & $45(86,5 \%)$ & $245(82,8 \%)$ & \\
\hline Sim & $7(13,2 \%)$ & $51(17,2 \%)$ & \\
\hline Dieta para emagrecer & & & 0,698 \\
\hline Não & $42(80,8 \%)$ & $232(78,4 \%)$ & \\
\hline Sim & $10(19,2 \%)$ & $64(21,6 \%)$ & \\
\hline Horas de sono/dia & & & 0,556 \\
\hline Menos de seis horas & $19(36,5 \%)$ & $121(40,9 \%)$ & \\
\hline Mais de seishoras & $33(63,5 \%)$ & $175(59,1 \%)$ & \\
\hline
\end{tabular}

\begin{tabular}{l|l} 
REVISTA BRASILEIRA DE EDUCAÇÃ̃ MÉDICA \\
\hline 44 (1) : e036; 2020
\end{tabular} 


\begin{tabular}{|c|c|c|c|}
\hline \multicolumn{4}{|c|}{$\begin{array}{c}\text { Tabela } 1 \\
\text { Continuação }\end{array}$} \\
\hline $\begin{array}{c}\text { Características e hábitos dos } \\
\text { estudantes }\end{array}$ & $\begin{array}{l}\text { Curso pré- } \\
\text { vestibular }\end{array}$ & Graduação & $\mathbf{P}$ \\
\hline Qualidade do sono & & & 0,803 \\
\hline Boa & $23(44,2 \%)$ & $144(48,6 \%)$ & \\
\hline Regular & $25(48,1 \%)$ & $134(45,3 \%)$ & \\
\hline Ruim & $4(7,7 \%)$ & $18(6,1 \%)$ & \\
\hline Uso de medicações diárias & & & $0,010^{*}$ \\
\hline Não & $31(59,6 \%)$ & $227(76,7 \%)$ & \\
\hline Sim & $21(40,4 \%)$ & $69(23,3 \%)$ & \\
\hline Uso de medicações para dormir & & & $<0,001^{*}$ \\
\hline Não & $41(78,8 \%)$ & $282(95,3 \%)$ & \\
\hline Sim & $11(21,2 \%)$ & $14(4,7 \%)$ & \\
\hline Uso de psicoestimulante & & & $0,001^{*}$ \\
\hline Não & $13(25,0 \%)$ & $148(50,0 \%)$ & \\
\hline Sim & $39(75,0 \%)$ & $148(50,0 \%)$ & \\
\hline
\end{tabular}

${ }^{*}$ Resultado significativo pelo teste qui-quadrado $(\mathrm{x} 2)(\mathrm{p}<0,05)$.

Fonte: Elaborada pelos autores.
Em relação ao tipo de estimulante utilizado em cada grupo, houve uma maior prevalência do uso atual de cafeína $(63,5 \%, \mathrm{p}=0,001)$ e de pó de guaraná $(11,5 \%, \mathrm{p}=0,040)$ entre os estudantes de pré-vestibular, e de ecstasy $(1,7 \%, \mathrm{p}=0,001)$ e metilfenidato $(3 \%, \mathrm{p}<0,001)$ entre os estudantes de ensino superior(Tabela 2).

Dentre os efeitos observados pelos usuários de estimulantes cerebrais do ensino superior, a redução do sono foi o efeito mais percebido $(64,9 \%, \mathrm{p}<0,001)$, seguido de melhora na concentração (48\%, p = 0,000), bem-estar $(45,3 \%, \mathrm{p}<0,001)$, raciocínio $(38,5 \%, \mathrm{p}$ $<0,001)$, redução da fadiga $(33,1 \%, \mathrm{p}<0,001)$, melhora na memória $(23,6 \%, p=0,002)$ e redução do estresse $(23 \%, p<0,001)$. Já nos estudantes do pré-vestibular, apenas a melhora no raciocínio $(43,6 \%$, $\mathrm{p}=0,018)$ e a redução do estresse $(28,2 \%, \mathrm{p}=0,031)$ obtiveram relevância significativa (Tabela 3 ).

A análise da Tabela 4 demonstra que, dentro do grupo dos prévestibulandos, $100 \%(\mathrm{n}=10, \mathrm{p}=0,042)$ que faziam regime para emagrecer eram usuários de algum tipo de estimulante cerebral. Já entre os acadêmicos, notou-se que a maior parte $(61,8 \%, \mathrm{n}=42, \mathrm{p}=$ $0,027)$ dos que fumam ou já fumaram fazia uso de psicoestimulantes. As demais variáveis avaliadas em cada grupo: tomar café da manhã, praticar

\begin{tabular}{|c|c|c|c|c|c|c|c|}
\hline Psicoestimulantes & Nunca ouviu falar & $\begin{array}{l}\text { Nunca usou ou } \\
\text { tem interesse }\end{array}$ & $\begin{array}{c}\text { Nunca usou, mas } \\
\text { se interessa }\end{array}$ & $\begin{array}{l}\text { Já usou algumas } \\
\text { vezes no passado }\end{array}$ & $\begin{array}{c}\text { Usava } \\
\text { regularmente no } \\
\text { passado }\end{array}$ & Usa atualmente & $\mathbf{P}$ \\
\hline Cafeína & & & & & & & $0,001^{*}$ \\
\hline Pré-vestibular & $0(0 \%)$ & $5(9,6 \%)$ & $5(9,6 \%)$ & $3(5,8 \%)$ & $6(11,5 \%)$ & $33(63,5 \%)$ & \\
\hline Graduação & $5(1,7 \%)$ & $79(26,7 \%)$ & $13(4,4 \%)$ & $60(20,3 \%)$ & $19(6,4 \%)$ & $120(40,5 \%)$ & \\
\hline Energético & & & & & & & 0,993 \\
\hline Pré-vestibular & $1(1,9 \%)$ & $12(23,1 \%)$ & $4(7,7 \%)$ & $25(48,1 \%)$ & $4(7,7 \%)$ & $6(11,5 \%)$ & \\
\hline Graduação & $5(1,7 \%)$ & $71(24 \%)$ & $23(7,8 \%)$ & $135(45,6 \%)$ & $19(6,4 \%)$ & $43(14,5 \%)$ & \\
\hline Pó de guaraná & & & & & & & $0,040^{*}$ \\
\hline Pré-vestibular & $1(1,9 \%)$ & $19(36,5 \%)$ & $10(19,2 \%)$ & $12(23,1 \%)$ & $4(7,7 \%)$ & $6(11,5 \%)$ & \\
\hline Graduação & $11(3,7 \%)$ & $163(55,1 \%)$ & $39(13,2 \%)$ & $65(22 \%)$ & $12(4,1 \%)$ & $6(2 \%)$ & \\
\hline Anfetamina & & & & & & & 0,051 \\
\hline Pré-vestibular & $20(38,5 \%)$ & $28(53,8 \%)$ & $3(5,8 \%)$ & $1(1,9 \%)$ & $0(0 \%)$ & $0(0 \%)$ & \\
\hline Graduação & $60(20,3 \%)$ & $217(73,3 \%)$ & $11(3,7 \%)$ & $7(2,4 \%)$ & $0(0 \%)$ & $1(3 \%)$ & \\
\hline Metilfenidato & & & & & & & $<0,001^{\star}$ \\
\hline Pré-vestibular & $4(7,7 \%)$ & $25(48,1 \%)$ & $16(30,8 \%)$ & $5(9,6 \%)$ & $1(1,9 \%)$ & $1(1,9 \%)$ & \\
\hline Graduação & $74(25 \%)$ & $177(59,8 \%)$ & $25(8,4 \%)$ & $14(4,7 \%)$ & $5(1,7)$ & $1(3 \%)$ & \\
\hline Ecstasy & & & & & & & $0,001^{*}$ \\
\hline Pré-vestibular & $17(32,7 \%)$ & $25(48,1 \%)$ & $3(5,8 \%)$ & $7(13, \%)$ & $0(0,0 \%)$ & $0(0,0 \%)$ & \\
\hline Graduação & $48(16,2)$ & $218(73,6 \%)$ & $10(3,4 \%)$ & $12(4,1 \%)$ & $3(1 \%)$ & $5(1,7 \%)$ & \\
\hline Piracetan & & & & & & & 0,135 \\
\hline Pré-vestibular & $35(67,3 \%)$ & $16(30,8 \%)$ & $1(1,9 \%)$ & $0(0,0 \%)$ & $0(0,0 \%)$ & $0(0,0 \%)$ & \\
\hline Graduação & $149(50,3 \%)$ & $142(48 \%)$ & $4(1,4 \%)$ & $0(0,0 \%)$ & $0(0,0 \%)$ & $0(0,0 \%)$ & \\
\hline Modafinil & & & & & & & 0,832 \\
\hline Pré-vestibular & $37(71,2 \%)$ & $14(26,9 \%)$ & $1(1,9 \%)$ & $0(0,0 \%)$ & $0(0,0 \%)$ & $0(0,0 \%)$ & \\
\hline Graduação & $202(68,2 \%)$ & $89(30,1 \%)$ & $3(1.0 \%)$ & $2(0,7 \%)$ & $0(0,0 \%)$ & $0(0,0 \%)$ & \\
\hline
\end{tabular}

* Resultado significativo pelo teste qui-quadrado $(\mathrm{x} 2)(\mathrm{p}<0,05)$.

Fonte: Elaborada pelos autores. 
atividade física, usar suplementos para ganho de massa muscular, horas de sono por dia, qualidade do sono, uso de medicações diárias, uso de medicações para dormir, curso pretendido, tempo no pré-vestibular, outros cursos realizados, graduação, período do curso, se já foi reprovado, carga horária do curso, turno do curso e atividades extracurriculares não apresentaram diferença estatística significativa em relação ao uso ou não de psicoestimulantes.

\section{DISCUSSÃO}

A atual prevalência do uso é maior entre os pré-vestibulandos (75\%) quando comparados aos universitários (50\%). Em contrapartida, o uso de psicoestimulantes seria mais comum em estudantes universitários, já que a maior parte deles iniciou o uso após entrar na faculdade, com o objetivo de aprimorar o desempenho acadêmico ${ }^{10}$. Supõe-se que os resultados encontrados no presente estudo, que evidenciou um maior uso por parte

\section{Tabela 3}

\begin{tabular}{|c|c|c|c|c|}
\hline \multicolumn{5}{|c|}{$\begin{array}{l}\text { Tabela } 3 \\
\text { ios de psicoestimulantes em cada grupo de estudantes avaliado }\end{array}$} \\
\hline Efeitos percebidos & Pré-vestibular & $\mathbf{p}$ & Graduação & $\mathbf{p}$ \\
\hline Melhora na concentração & & 0,071 & & $<0,001^{\star}$ \\
\hline Não & $8(20,5 \%)$ & & $77(52 \%)$ & \\
\hline Sim & $31(79,5 \%)$ & & $71(48 \%)$ & \\
\hline Melhora na memória & & 0,355 & & $0,002^{*}$ \\
\hline Não & $28(71,8 \%)$ & & $113(76,4 \%)$ & \\
\hline Sim & $11(28,2 \%)$ & & $35(23,6 \%)$ & \\
\hline Melhora no raciocínio & & $0,018^{\star}$ & & $<0,001^{\star}$ \\
\hline Não & $22(56,4 \%)$ & & $91(61,5 \%)$ & \\
\hline Sim & $17(43,6 \%)$ & & $57(38,5 \%)$ & \\
\hline Melhora no bem-estar & & 0,105 & & $<0,001^{\star}$ \\
\hline Não & $20(51,3 \%)$ & & $81(54,7 \%)$ & \\
\hline Sim & $19(48,7 \%)$ & & $67(45,3 \%)$ & \\
\hline Redução do sono & & 0,377 & & $<0,001^{\star}$ \\
\hline Não & $10(25,6 \%)$ & & $52(35,1 \%)$ & \\
\hline Sim & $29(74,4 \%)$ & & $96(64,9 \%)$ & \\
\hline Redução do estresse & & $0,031^{*}$ & & $<0,001^{*}$ \\
\hline Não & $28(71,8 \%)$ & & $114(77 \%)$ & \\
\hline Sim & $11(28,2 \%)$ & & $34(23 \%)$ & \\
\hline Redução da fadiga & & 0,868 & & $<0,001^{\star}$ \\
\hline Não & $25(64,1 \%)$ & & $99(66,9 \%)$ & \\
\hline Sim & $14(36,5 \%)$ & & $49(33,1 \%)$ & \\
\hline
\end{tabular}

* Resultado significativo pelo teste qui-quadrado $(\mathrm{x} 2)(\mathrm{p}<0,05)$.

Fonte: Elaborada pelos autores.

\begin{tabular}{|c|c|c|c|c|c|c|}
\hline \multicolumn{7}{|c|}{$\begin{array}{l}\text { Tabela } 4 \\
\text { Variáveis associadas ao uso de psicoestimulantes em cada grupo de estudantes avaliado }\end{array}$} \\
\hline \multirow{3}{*}{ Variável } & \multirow{2}{*}{\multicolumn{2}{|c|}{$\begin{array}{l}\text { Pré-vestibular } \\
\text { Usuário }\end{array}$}} & \multirow{2}{*}{\multicolumn{3}{|c|}{$\begin{array}{c}\text { Graduação } \\
\text { Usuário }\end{array}$}} & \multirow{3}{*}{$\mathbf{p}$} \\
\hline & & & & & & \\
\hline & Não & Sim & & Não & Sim & \\
\hline Dieta para emagrecer & & & $0,042^{*}$ & & & 0,572 \\
\hline Não & $13(100 \%)$ & $29(74,4 \%)$ & & $114(77 \%)$ & $30(20,3 \%)$ & \\
\hline Sim & $0(0,0 \%)$ & $10(25,6 \%)$ & & $34(23 \%)$ & $118(79,7 \%)$ & \\
\hline Fuma ou já fumou? & & & 0,071 & & & $0,027^{*}$ \\
\hline Não & $12(92,3 \%)$ & $26(66,7 \%)$ & & $122(82,4 \%)$ & $106(71,6 \%)$ & \\
\hline Sim & $1(7,7 \%)$ & $13(33,3 \%)$ & & $26(17,6 \%)$ & $42(28,4 \%)$ & \\
\hline
\end{tabular}

${ }^{*}$ Resultado significativo pelo teste qui-quadrado (x2) $(\mathrm{p}<0,05)$.

Fonte: Elaborada pelos autores. 
dos pré-vestibulandos, estejam relacionados ao fato de estes estarem submetidos a uma maior pressão, o que motivaria a estudar mais para disputar uma vaga no ensino superior e utilizar drogas psicoativas para alcançar o seu objetivo ${ }^{11}$.

Pode-se correlacionar o uso de drogas lícitas e ilícitas entre estudantes com as dificuldades encontradas durante os anos de estudo, além dos conflitos afetivos e das inseguranças relacionadas ao futuro. $\mathrm{O}$ fator ambiental é determinante para a utilização de diversas drogas, uma vez que, quando o estudante busca um tipo de substância entorpecente, cria-se um meio favorável para o uso de outras ${ }^{12}$.

Dentre as substâncias pesquisadas, observou-se maior consumo de estimulantes naturais como cafeína e pó de guaraná entre os prévestibulandos. Provavelmente, o maior consumo de cafeína ocorre por mais fácil acesso à substância e do baixo custo para adquiri-la. $\mathrm{O}$ pó de guaraná tem como principal composto a guaranina, proporciona, como a cafeína, picos energéticos e também é bastante utilizado por estudantes. Um estudo mostra que esse composto é menos prevalente, no entanto, que a cafeína e os energéticos ${ }^{6}$.

Observou-se uma maior associação entre os pré-vestibulandos que fazem uso de cafeína e pó de guaraná e realização de dieta. Uma possível correlação com esse dado é que tais substâncias psicoativas estimulam o metabolismo e suprimem o apetite, facilitando a perda do peso corporal por conta de uma elevação da termogênese, lipólise e oxidação de gordura por mecanismos pouco compreendidos ${ }^{13}$. O uso de drogas estimulantes estaria associado também a um propósito de emagrecimento por parte dos estudantes que delas fazem uso, uma vez que a rotina atarefada dificulta a prática de atividades físicas e uma alimentação adequada, favorecendo o ganho peso e a obesidade ${ }^{14}$.

Dentre os efeitos observados no presente estudo, a melhora no raciocínio $(43,6 \%)$ e a redução no estresse $(28,2 \%)$ foram os que obtiveram maior relevância estatística entre os estudantes do pré-vestibular. Corroborando esse fato, constatou-se que substâncias psicoativas melhoram o raciocínio, o que representa o principal estímulo para o uso de estimulantes cerebrais ${ }^{15}$.

Outros efeitos de potencialização das funções mentais, como melhora na concentração e na memória, bem-estar, além de redução de fadiga, não foram encontrados o que destoa de outros trabalhos. Em relação à redução no estresse, ao contrário do que foi encontrado, o uso dessas substâncias está associado à diminuição de qualidade de vida por causa de um aumento do estresse, o que pode acarretar inclusive uma elevação na morbidade dos estudantes'.

Em relação aos universitários, os resultados mostraram que houve uma experiência de uso relativamente maior de metilfenidato e ecstasy, consolidando que os psicotrópicos são consumidos com o propósito de aumentar a capacidade cerebral em atividades acadêmicas ${ }^{15}$. O cloridrato de metilfenidato - um estimulante cerebral que age na inibição de noradrenalina e dopamina - é um dos psicotrópicos mais utilizados. Entretanto, um estudo demonstra que há um crescimento do uso de ecstasy por universitários nas últimas décadas ${ }^{17}$.

O maior consumo de drogas ilícitas entre estudantes é uma questão de saúde pública, estando relacionado a agressões, comportamentos sexuais de risco, acidentes automobilísticos e alterações de comportamento ${ }^{18}$. Todavia, deve-se destacar que houve apenas cinco casos entre os universitários, o que pode ter ocorrido pelo fato de a amostra desses estudantes ter sido maior que a dos estudantes de pré-vestibular, favorecendo o resultado estatístico obtido na pesquisa.

O metilfenidato e o ecstasyaumentam a concentração, reduzem o sono e a fadiga e melhoram a cognição e a coordenação motora ${ }^{19,20}$. Esses efeitos positivos foram encontrados no presente estudo que constatou redução do sono (64,9\%), melhora na concentração (48\%), no raciocínio (38,5\%), redução da fadiga $(33,1 \%)$ e melhora da memória $(23,6 \%)$. Os principais efeitos colaterais do metilfenidato são a redução do apetite, insônia, hipertensão arterial, além de poder causar cefaleia e dor abdominal ${ }^{21}$. Em relação ao ecstasy, osefeitos observados em curto prazo são: euforia, insônia, humor deprimido, diminuição da ansiedade, alterações na cognição, psicose e alucinações ${ }^{22}$.

$\mathrm{Na}$ amostra geral, constataram-se uma pior qualidade de sono e altos índices de uso de medicações indutoras do sono (72,7\%), o que pode ser justificado com base em uma formulação que infere que os usuários de psicoativos têm uma perturbação do sono que altera a cognição e a funcionalidade física e social. Além disso, esse tipo de medicamento reduz a qualidade de vida dos usuários. Dessa forma, supõe-se que esses indivíduos fazem uso de indutores do sono com a finalidade de reverter os efeitos colaterais de tais drogas, melhorando o ciclo sono-vigília ${ }^{23}$.

Não foram encontradas diferenças significativas quanto ao uso das substâncias psicoativas pesquisadas nas diferentes áreas do ensino superior (Direito, Engenharia e Medicina). Por conta de uma maior exigência no meio acadêmico, tais estudantes utilizam o estimulante de forma indiscriminada para reduzir o cansaço cotidiano, de modo a elevar o rendimento e a concentração nos estudos, além da cognição, independentemente de estarem vinculados às Ciências Exatas, Biológicas ou Humanas ${ }^{24,25,26}$

É importante ressaltar que, para as análises do presente estudo, apenas os indivíduos que estavam em uso no momento da pesquisa foram classificados como usuários de psicoestimulantes, diferentemente de outros estudos que consideraram usuário aquele que já fez uso em alguma época na vida. Tal situação dificulta a comparação entre dados da literatura, mas reafirma o problema apresentado de uma forte exposição precoce de jovens a tais substâncias. Além disso, o caráter transversal da pesquisa também dificulta o estabelecimento de relações de causa, efeito e temporalidade das variáveis estudadas, as quais são importantes para a identificação de situações que promovessem riscos aumentados à população investigada.

A maioria da amostra relatou uma eficácia no uso das substâncias pesquisadas, o que faz com que haja uma alta ocorrência do uso contínuo delas e uma maior dificuldade na descontinuação do uso. Porém, sabese que a utilização em longo prazo é nociva à saúde, já que pode gerar dependência e tolerância. Nesse caso, os indivíduos sentem necessidade de utilizar quantidades cada vez maiores dessas substâncias e de outras drogas, o que pode prejudicar os estudantes nas práticas acadêmicas e laborais futuras. Além disso, o uso continuo está associado a efeitos cardiovasculares e a uma possível redução de estatura, como é caso do metilfenidato ${ }^{21,26}$. Diante disso, reforça-se a necessidade de mais pesquisas associadas ao tema abordado, visando reduzir os efeitos adversos, estipular uma quantidade segura e limitar o uso de certas substâncias.

É sempre importante ressaltar os malefícios do uso de psicoestimulantesem longo prazo, sobretudo a dependência e a tolerância química, fatores que predispõem o indivíduo a consumir 
quantidades cada vez maiores dessas substâncias. O metilfenidato é uma droga que, além de poder ocasionar dependência, gera reações adversas em todo o organismo, principalmente no sistema nervoso central $^{27}$. Em relação à cafeína, psicoestimulante mais utilizado pelos estudantes, há relatos de que doses elevadas (concentração plasmática maior que $50 \mu \mathrm{M}$, o que equivale a, no mínimo, cinco e, no máximo, dez xícaras ${ }^{28}$ ) podem promover tolerância, sendo comum a ocorrência de sensação de angústia, nervosismo e agitação ${ }^{29}$. Como consequência direta do uso contínuo e demasiado de psicoestimulantes, frequentemente se observa, além dos malefícios relacionados à saúde, o surgimento de problemas psicológicos, sociais, financeiros e acadêmicos, condições que certamente conturbarão a vida do estudante.

$\mathrm{Na}$ tentativa de prevenir as consequências danosas do uso prolongado e abusivo de psicotrópicos, é fundamental que o estudante receba apoio familiar e psicopedagógico. $\mathrm{O}$ uso de psicotrópicos entre estudantes é um assunto que não envolve apenas questões sociais, mas também a própria instituição de ensino, que, muitas vezes, não proporciona mecanismos protetores contra o uso dessas substâncias ${ }^{30}$. Há ainda algumas medidas que podem ajudar na diminuição do uso não prescrito de psicoestimulantes, como criar meios dentro da instituição que ajudem a reduzir o estresse vivenciado pelos alunos, promover a conscientização de alunos e professores sobre a importância do tema em nosso meio e permitir debates acerca do uso indiscriminado de drogas do ponto de vista biopsisicossocial ${ }^{30}$. Os familiares podem buscar auxílio de profissionais habilitados que, por meio dos grupos de apoio, passarão orientações para melhor enfrentar o caminho da recuperação. $\mathrm{O}$ apoio psicopedagógico também pode avaliar anormalidades no comportamento dos estudantes e identificar as causas do insucesso acadêmico, para posteriormente implementar medidas de correção das dificuldades encontradas, mediante averiguação, intervenção e acompanhamento.

\section{CONCLUSÃO}

Observou-se uma maior prevalência do uso de psicoestimulantes nos pré-vestibulandos em relação ao grupo dos universitários, sendo a cafeína e o pó de guaraná os mais utilizados. Entre os pré-vestibulandos, houve uma significativa maior realização de dieta e uso de medicações indutoras do sono. Entre os universitários, houve uso de metilfenidato e ecstasy. Foram relatados efeitos como redução do sono, melhora na concentração, no raciocínio, redução da fadiga e melhora da memória. Nas áreas do ensino superior (Direito, Engenharia e Medicina), não se constataram diferenças quanto ao uso dos estimulantes cerebrais pesquisados.

É notável que habitualmente sempre haverá alguma associação entre fatores sociais e ambientais que expliquem o uso de estimulantes cerebrais por parte dos estudantes. Portanto, o apoio familiar e o psicopedagógico são indispensáveis para prevenir e tratar as consequências do uso desmedido de psicoestimulantes.

\section{REFERÊNCIAS}

1. Peuker ACWB, Fogaça JL, Araujo LB. Expectativas e beber problemático entre universitários. Psicol. teor. pesqui. 2006;22(2):193200.

2. Pope JR, Harrison G, Ionescu-Pioggia M, Pope KW. Drug use and life style among college undergraduates: a 30-year longitudinal study. Am: j. psychiat. 2001;158(9):1519-21.
3. Almeida LS, Soares AP. Os estudantes universitários: sucesso escolar e desenvolvimento psicossocial. In: Mercuri E, Polydoro SAJ, organizadores. Estudante universitário: características e experiências de formação. Taubaté: Cabral Editora e Livraria Universitária; 2004. p.15-40.

4. Lemos KM, Neves NMBC, Kuwanol AY, Tesdesquil G, Bitencourt AGV, Neves FBCS, et al. Uso de substâncias psicoativas entre estudantes de Medicina de Salvador (BA). Rev. Psiquiatr. clínic. 2007;34(3):118-24.

5. Kumar R. Approved and investigational uses of modafinil: an evidence-based review. Drugs 2008;68(13):1803-39.

6. Silveira VI, Oliveira RJF, Caixeta MR, Andrade BBP, Costa RGL, Santos GB. Uso de psicoestimulantes por acadêmicos de Medicina de uma universidade do sul de Minas Gerais. Revista da Universidade Vale do Rio Verde 2015;13(2):186-92.

7. Cesar ELR, Wagner GA, Castaldelli-Maia JM, Silveira CM, Andrade AG, Oliveira LG. Uso prescrito de cloridrato de metilfenidato e correlatos entre estudantes universitários brasileiros. Arch. Clin. Psychiatry 2012;39(6):183-8.

8. Pasquini NC. Uso de metilfenidato (MFD) por estudantes universitários com intuito de "turbinar" o cérebro. Revista Brasileira de Biologia e Farmácia 2013;9(2):107

9. Morgan HL, Petry AF, Licks PAK, Ballester AO, Teixeira KN, Dumith SC. Consumo de estimulantes cerebrais por estudantes de medicina de uma universidade do extremo sul do brasil: prevalência, motivação e efeitos percebidos. Rev. bras. educ. méd. 2017;41(1):102-9.

10. Finger G, Silva ERD, Falavigna A. Use of methylphenidate among medical students: a systematic review. Rev. Assoc. Med. Bras. 2013;59(3):285-9.

11. Shah M, Hasan S, Malik S, Sreeramareddy C. Perceived stress, sources and severity of stress among medical undergraduates in a Pakistani medical school. BMC med. educ. 2010; 10(1):2.

12. Trindade BPA, Diniz AV, Sá-Júnior AR. Uso de drogas entre estudantes universitários: uma perspectiva nacional. Revista de Medicina e Saúde de Brasília 2018;7(1):52-60.

13. Lopez-Garcia E, Van Dam RM, Rajpathak S, Willett WC, Manson JE, HU FB. Changes in caffeine intake and long-term weight change in men and women. The American Journal of Clinical Nutrition 2006;83(3):674-80.

14. Mota MC, Souza DAD, Mello MTD, Tufik S, Crispim CA. Estilo de vida e formação médica: impacto sobre o perfil nutricional. Rev. bras. educ. méd. 2012;36(3):358-8.

15. Chatterjee A. Cosmetic neurology: the controversy over enhancing movement, mentation, and mood. Neurology 2004;63(6):968-74.

16. Coli ACM, Sousa MP, Nakasu MVP. Uso não prescrito de metilfenidato entre estudantes de uma faculdade de medicina do sul de Minas Gerais. Rev. Ciênc. Saúde 2016;6(3):121-32.

17. Mendes SV, Troncoso LDT, Nascimento BS, Mühlbauer M. Estudo sobre o uso de drogas estimulantes entre estudantes de Medicina. Ciência Atual - Revista Científica Multidisciplinar das Faculdades São José 2015;5(1):2-6.

18. Zeferino MT, Hamilton H, Brands B, Wright MDGM, Cumsille F, Khenti A. Consumo de drogas entre estudantes universitários: família, espiritualidade e entretenimento moderando a influência dos pares. Texto \& contexto enferm. 2015;24:125-35.

7 REVISTA BRASILEIRA DE EDUCAÇÃO MÉDICA

7 74 (1) : e036; 2020 
19. Andrade ÊRD, Scheuer C. Analysis of the methylphenidate's efficacy using the abbreviated version Conners' questionnaire in attention deficit hyperactivity disorder. Arquivos de Neuro Psiquiatria 2004;62(1):81-5.

20. Green WH. Sympathomimetic amines and central nervous system stimulants. In: Green WH. Child and adolescent clinical psychopharmacology. $4^{\text {th }}$ ed. Philadelphia: LWW;2007. p.55-90.

21. Pastura G, Mattos P. Efeitos colaterais do metilfenidato. Arch. Clin. Psychiatry 2004;31(2): 100-4.

22. Sjegel RK. MDMA nonmedical use and intoxication. J. Psycoactive Drugs 1986;18(4):349-54.

23. Cardoso HC, Bueno FC, Mata JC, Alves AP, Jochims I, Filho IHV, Hanna MM. Avaliação da qualidade do sono em estudantes de Medicina. Rev. bras. educ. méd. 2009;33(3):349-55.

24. Castilho CP, Limas LMD, Monteiro ML, Silva PHMN, Bueno H, Fari TA. A privação de sono nos alunos da área de saúde em atendimento nas Unidades Básicas de Saúde e suas consequências. Revista de Medicina 2015;94(2):1139.

25. Cruz TCSC, Barreto Junior EPS, Gama MLM, Maia LCM, Filho MJXM, Neto OM, Coutinho DM. Uso não-prescrito de metilfenidato entre estudantes de Medicina da Universidade Federal da Bahia. Gazeta Médica da Bahia 2011;81(1):3-6.

26. Marcon C, Silva MAL, Moraes BMC, Martins SJ, Carpes DA. Amphetamines use and related substances in contemporary society. Disciplinarum Scientia 2012;13(2):247-63.

27. Smith ME, Farah MJ. Are prescription simulants "smart pills"? The epidemiology and cognitive neuroscience of prescription simulant use by normal healthy individuals. Psychol. bull. 2011;137(5):717.
28. Wong DWS. Química de los alimentos - mecanismos y teoria. Zaragoza: Editorial Acribia; 1995. p.318-9.

29. Ramos WPB. Abuso de drogas. In: Silva P. Farmacologia. 8.ed. Rio de Janeiro: Guanabara Koogan; 2010.

30. Mesquita AMC, Bucaretchi HA, Castel S, Andrade AG. Estudantes da Faculdade de Medicina de São Paulo: uso de substâncias psicoativas em 1991. Rev. ABP-APAL 1995;17(2):47-54.

\section{CONTRIBUIÇÃO DOS AUTORES}

Todos os autores atuaram na coleta de dados, análise, construção e redação do artigo e aprovaram a versão final do manuscrito.

\section{CONFLITO DE INTERESSES}

Os autores declaram não ter nenhum conflito de interesses relativamente ao presente artigo.

\section{ENDEREÇO PARA CORRESPONDÊNCIA}

Luíza Côrtes Santana

Avenida Professora Aida Mainartina Paraíso, n 80. Ibituruna, Montes Claros - MG.

CEP: 39408-007.

E-mail: luiza.cortes@hotmail.com; andreza.nevesramos@gmail.com; bruna.lopes.95@hotmail.com; inacioluiz47@gmail.com; mateuslima19@ hotmail.com; mvmoliv@gmail.com. 